\title{
XMM-Newton witness of M 86 X-ray metamorphosis
}

\author{
A. Finoguenov ${ }^{1,3}$, W. Pietsch ${ }^{1}$, B. Aschenbach ${ }^{1}$, and F. Miniati ${ }^{2}$ \\ 1 Max-Planck Institut für extraterrestrische Physik, Giessenbachstraße 1, 85748 Garching, Germany \\ 2 Max-Planck Institut für Astrophysik, Karl-Schwarzschild Str. 1, 85748 Garching, Germany \\ 3 Space Research Institute, Profsoyuznaya 84/32, Moscow, 117810, Russia
}

Received 28 May 2003 / Accepted 15 October 2003

\begin{abstract}
The environmental influence of cluster media on its member galaxies, known as Butcher-Oemler effect, has recently been subject to revision due to numerous observations of strong morphological transformations occurring outside the cluster virial radii, caused by some unidentified gas removal processes. In this context we present new XMM-Newton observations of M 86 group. The unique combination of high spatial and spectral resolution and large field of view of XMM-Newton allows an in-depth investigation of the processes involved in the spectacular disruption of this object. We identify a possible shock with Mach number of $\sim 1.4$ in the process of crushing the galaxy in the North-East direction. The latter is ascribed to the presence of a dense X-ray emitting filament, previously revealed in the RASS data. The shock is not associated with other previously identified features of M 86 X-ray emission, such as the plume, the north-eastern arm and the southern extension, which are found to have low entropy, similar to the inner $2 \mathrm{kpc}$ of M 86. Finally, mere existence of the large scale gas halo around the M 86 group, suggests that the disruptions of M 86's X-ray halo may be caused by small-scale types of interactions such as galaxy-galaxy collisions.
\end{abstract}

Key words. galaxies: individual: M 86 - galaxies: interactions - galaxies: structure - ISM: kinematics and dynamics

\section{Introduction}

X-ray studies of galaxies in nearby clusters provide important clues on the action of environmental effects on the properties of galaxies. X-ray luminous early-type galaxies appear often surrounded by group-size mass concentrations, whose interaction with the cluster environment is not yet completely understood from both observational and modeling sides. In fact, on one hand, recent observations find quite a bit of substructure in merging systems, demonstrating the survival of the core of the accreting objects (e.g. Vikhlinin et al. 2001). On the other hand, detailed studies of cluster galaxies show that strong interactions take place among the infalling galaxies at the virial radius (Poggianti et al. 1999; Goto et al. 2003). In addition, recent studies on the evolution of galaxies prior to their infall onto clusters reveal strong effects of local environment on the appearance of galaxies (Kodama et al. 2001). Therefore, the morphology of galaxies appear to be disturbed in the so called quiet accretion mode of cluster formation.

M 86 is a prototype for this type of investigation. It is a giant elliptical galaxy falling at high speed toward M 87 (relative velocity $\sim 1500 \mathrm{~km} \mathrm{~s}^{-1}$ ). Furthermore it sits in the core of a larger structure, M 86 groups of galaxies, which exhibits a diffuse X-ray emission on a few hundreds kpc scale (Böhringer et al. 1994). An X-ray study of M 86 with Einstein

Send offprint requests to: A. Finoguenov, e-mail: alexis@xray.mpe.mpg.de
(Forman et al. 1979) revealed a peak of emission centered on M 86 and a plume extending northwest of the galaxy. Rampressure stripping of the hot gas from M 86 as the galaxy traverses the Virgo cluster was suggested to explain the observed structure (Forman et al. 1979; Fabian et al. 1980; Takeda et al. 1984). Spatial analysis of the X-ray brightness of M 86 from the ROSAT PSPC and HRI data was presented in Rangarajan et al. (1995). In addition to the plume, they identified a southern extension (extending from the center by about $3^{\prime}$ to the south), a void (north to the galaxy), and a northeastern arm (extending from the center by more than $5^{\prime}$ to the northeast). Detailed spectral analysis of M 86 emission using ROSAT PSPC and ASCA SIS results was presented in Finoguenov \& Jones (2000). The temperature variations were found to lie in the $0.6-1.1 \mathrm{keV}$ range. Cool regions include the galaxy center and extend eastward with slightly increasing temperature. In the plume, spectral variations were detected on the $0.1 \mathrm{keV}$ level.

As it became evident from the temperature and detailed surface brightness maps, M 86 does not fit into the simple picture suggested by early observations. For this reason Rangarajan et al. (1995) proposed that M 86 owes its appearance to the presence of a conical shock seen in projection and due to the galaxy interaction with Virgo intracluster medium (ICM).

In this paper we present new XMM-Newton observations of M 86 galaxy. By taking advantage of the unique combination of high spatial and spectral resolution of XMM-Newton, we study the thermodynamic properties of a total of 120 regions 

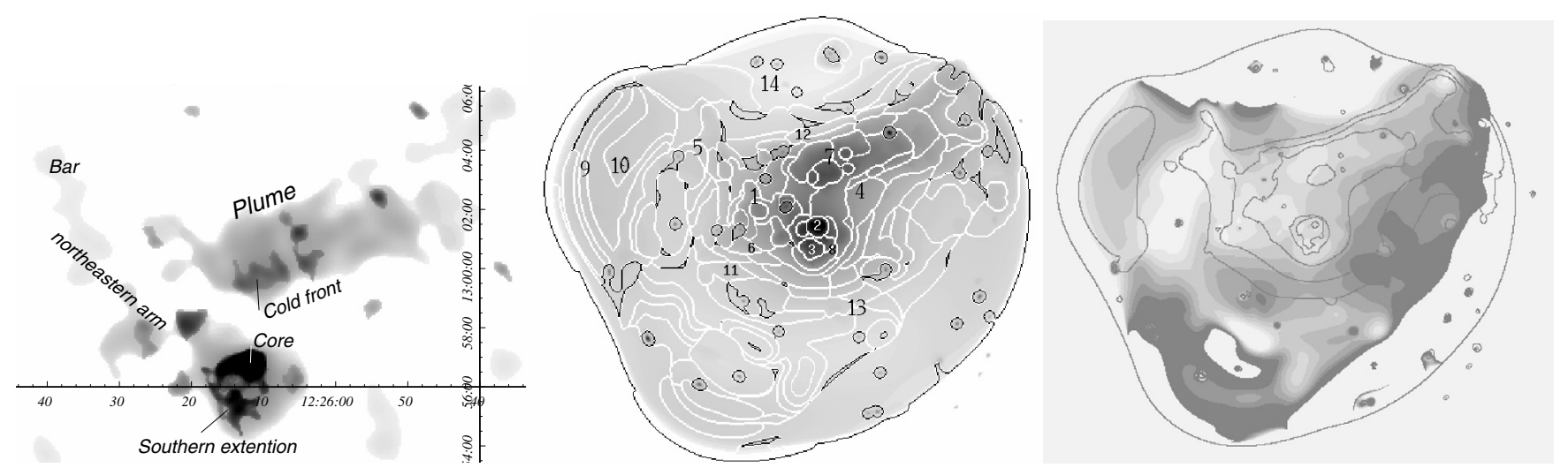

Fig. 1. Left panel. Identification of the previously reported features of M 86 on the smallest scale of the wavelet decomposition of the image in the 0.5-2 keV band. Middle panel. Wavelet-decomposed image of M 86 with contours indicating the selection of regions for the spectral analysis. Black contours denote the regions excluded from the analysis due to either low statistics or a presence of the point source. Numbers on the figure indicate the approximate location of the larger zones, reported in Table 1. Right panel. Hardness ratio map with surface brightness contours overlaid. Dark grey color corresponds to temperature of $1.1-1.2 \mathrm{keV}$, grey to $0.9-1 \mathrm{keV}$, light grey to $0.7-0.9 \mathrm{keV}$, white to $0.5-0.7 \mathrm{keV}$.

comprising the environment in and around $\mathrm{M} 86$. Based on these, we were able to reveal the remarkable presence a of a weak shock in the process of crushing the galaxy interstellar medium (ISM). In view of a $2.4 \pm 1.4 \mathrm{Mpc}$ distance of M 86 to the core of Virgo (Neilsen \& Tsvetanov 2000), it is, however, quite unlikely for such shock to be driven by the impact of the galaxy on Virgo ICM, even considering the uncertainties in the distance determination. A conclusion that M 86 group is located outside the M 87 cloud is further supported by observation of the X-ray halo extending over a degree in diameter (Böhringer et al. 1994). We have therefore carried out a closer inspection of the large scale gas distribution surrounding M 86 which led us to associate the counteracting structure with a filament in the neighborhood of Virgo cluster, already discovered by Böhringer et al. (1994).

The picture of M 86 system emerging from these observations argues for a series of interactions at different stages of completeness. Although the weak shock is probably driven by an interaction with the surrounding large scale medium, the large-scale emission of M 86 group is mostly unchanged. On the contrary, the core of M 86 galaxy has been already strongly perturbed. This suggests that additional significant dynamic interactions were responsible for disturbing the X-ray morphology of M 86 galaxy and that they took place on small scales, of order $10-50 \mathrm{kpc}$. Galaxy-galaxy encounters may offer a possible explanation.

The paper is organized as follows: in Sect. 2 we present a detailed description of the data, including the reconstruction of maps of thermodynamic quantities in Sect. 2.1 that lead to the identification of a weak shock in Sect. 2.2. The results are discussed in Sect. 3 and summarized in Sect. 4.

In the following we will assume a $19.5 \mathrm{Mpc}$ distance to M 86 (Neilsen \& Tsvetanov 2000), for which $1^{\prime}=5.67 \mathrm{kpc}$.

\section{Observations and data reduction}

M 86 was observed by XMM-Newton (Jansen et al. 2001) during July $1-2,2002$, as a part of the GTO program of the telescope scientists at MPE. Screening of the observations to remove particle background flaring episodes results in the net exposure time of $46 \mathrm{ks}, 64 \mathrm{ks}$ and $67 \mathrm{ks}$ for pn, MOS1 and MOS2 EPIC detectors. pn observations were performed with medium filter and in the extended full frame observing mode (Strüder et al. 2001), which is characterized by a reduced level of out-of-time events. MOS1 and MOS2 (Turner et al. 2001) observations were performed with the thick filter and in the present study are used only for the imaging analysis. The advantage of using MOS and RGS spectra is mostly in the detailed element abundance study (particularly given the thick filter choice for the MOS and a typical temperature of the X-ray emission of M 86), which will be given in a separate paper.

Initial steps of data reduction were performed using XMMSAS 5.4. The spectral analysis of pn data was carried out in the $0.4-5 \mathrm{keV}$ band. For the background subtraction we used several background accumulations, one by Read (2003) and the other performed close to the observation (e.g. APM08279+5255; Hubble Deep Field South). We find that for the M 86 observation, the detector background in the $10-15 \mathrm{keV}$ range corresponds better to observations performed at similar epochs. Although the details of the background subtraction are not very critical for the analysis of the soft emission of M 86, it could be of some importance for the analysis of the hard component, associated with unresolved LMXBs.

The vignetting correction is performed taking the source extent and a recent vignetting calibration (Lumb et al. 2003) into account, which is mostly important for the absolute flux determination, given our choice of the energy range. Remaining systematic uncertainty of the flux is below $4 \%$ for both pn and MOS (Lumb et al. 2003).

In selecting the regions for the spectral analysis we use a combination of the surface brightness and hardness ratio maps to emphasize regions of similar color and intensity. Figure 1 shows both maps, where the surface brightness is a waveletreconstructed (Vikhlinin et al. 1998) image in the $0.5-2 \mathrm{keV}$ band and the hardness of the emission is a ratio of the wavelet-reconstructed images in the $0.5-1$ and $1-2 \mathrm{keV}$ bands. 

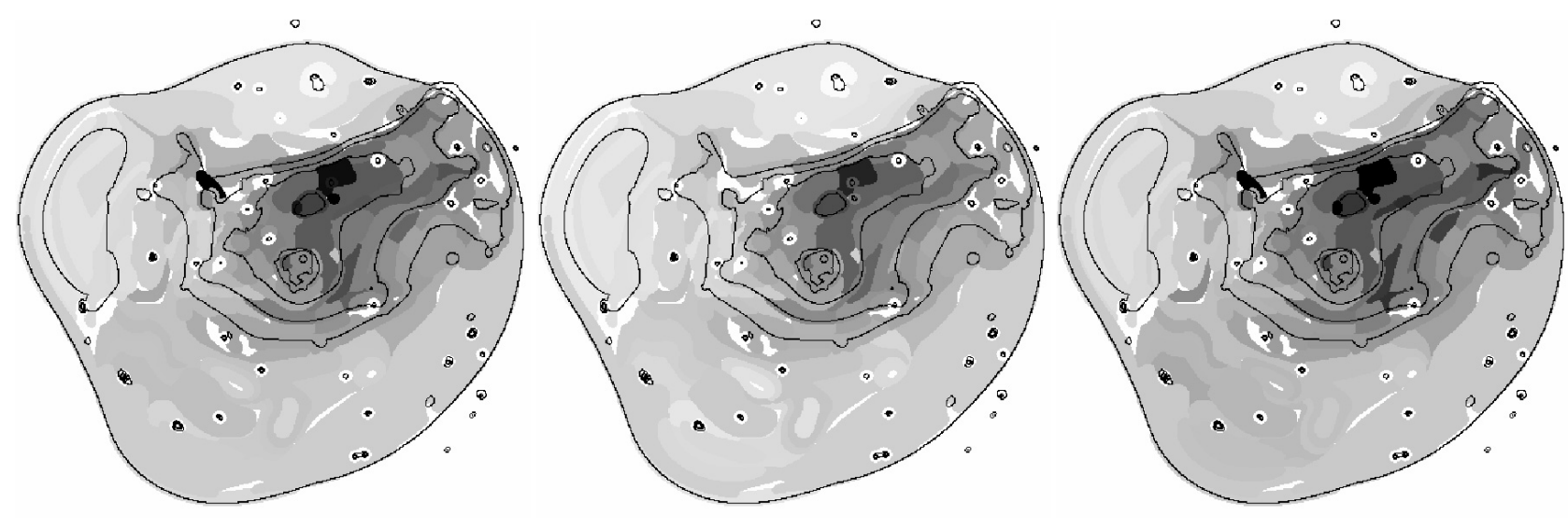

Fig. 2. Fe abundance map, overlaid with surface brightness contours. From left to right the panels show the best-fit values, low and upper limit on Fe abundance, ranging from 0 (light grey) to 0.7 (black) of solar.

An advantage of using wavelets consists in background removal by spatial filtering and a control over the statistical significance of the detected structure. Complications arise due to splitting the image into discrete scales, which we overcome by additional smoothing applied before producing the hardness ratio map. More details can be found in Briel et al. (2003). In combining MOS and pn, we differentially normalize the exposure maps to account for the difference in the sensitivity of MOS vs. pn to the temperatures in the $0.6-1.2 \mathrm{keV}$ range, given our selection of bands. In doing so, we avoid variations in color due to different sky coverage provided by MOS and pn. Given the choice of the energy bands, variation in the $\mathrm{Fe}$ abundance should not play a strong role in the color selection, however iron line emission determines up to $90 \%$ of the flux in the $0.5-2 \mathrm{keV}$ band. To establish the mask file for the further spectral analysis we use the changes in the hardness ratio that correspond to temperature in the ranges $0.48-0.56-0.60-0.64$ $0.72-0.8-0.9-1.0-1.1-1.2 \mathrm{keV}$ and have equal intensity within a factor of two. Taking the isolated regions of equal color and intensity separately and imposing the criterion that the regions should be larger than the PSF (15") width and contain more than 1000 counts in the raw pn image, we obtain the final mask used in the spectral extraction, which we display as contours in the central panel of Fig. 1.

For the spectral fits, we first assume a single temperature plasma in collisional ionization equilibrium, with emission due to thermal bremsstrahlung dominated by $\mathrm{H}, \mathrm{He}, \mathrm{C}$ and $\mathrm{O}$ ions and line emission which, at the temperature typical of M 86 and in the energy range of XMM-Newton, is dominated by $\mathrm{Fe}$ (e.g. Raymond \& Smith 1977). For the purpose we use the recent revision of the initial Raymond-Smith code, APEC (Smith et al. 2001), implemented in the XSPEC v.11.2, which benefits from improved treatment of the Fe L-shell line atomic physics (e.g. Liedahl et al. 1995). In the spectral analysis, we leave temperature and abundances of $\mathrm{O}, \mathrm{Ne}, \mathrm{Mg}, \mathrm{Si}, \mathrm{Fe}$ as free parameters. $\mathrm{C}$ abundance, which cannot be fit, but contributes to the continuum emission is set to 0.2 times the solar value. Setting $\mathrm{C} / \mathrm{O}$ ratio to solar gives the same results.

As a second step, a power law component which, according to several studies well describes the emission from low mass X-ray binaries (LMXB) (Finoguenov \& Jones 2001, 2002;
Irwin et al. 2003) was introduced. We need to do so, owing to high level of diffuse X-ray emission of M 86 and the PSF of the XMM telescopes, which prevent us from resolving LMXB individually. The spectral index found by fitting the regions close to the M 86 core and is 1.5 , consistent with the results for other galaxies (Irwin et al. 2003). In the following we fix the slope and only fit the normalization of the model.

Finally few regions close to the center of M 86 still require a more complex spectral model, which we achieve by adding a second plasma (APEC) component. Since the emission weighted temperature has not changed within estimated statistical errors, we conclude that the results from the previous steps on temperature structure are still valid. In all the fits $N_{\mathrm{H}}$ was fixed at the Galactic value of $2.7 \times 10^{20} \mathrm{~cm}^{-2}$ (Stark et al. 1992).

\subsection{Maps}

The spectral analysis provides statistically significant data on $k T$ for all of the 120 selected regions. Metal abundances and normalizations are well constrained for $\mathrm{Fe}$ in most of those regions, for $\mathrm{Mg}$ and $\mathrm{Si}$ for some of them, and for $\mathrm{O}$ and $\mathrm{Ne}$ in only a few of them. In Figs. 2 and 3 we show the temperature and iron abundance maps. We find temperature variations in the $0.6-1.2 \mathrm{keV}$ range, in general agreement with the previous findings. In addition several new details are revealed by these maps: the northeastern arm, for example, is clearly seen as a part of the cold spiraling thread starting from the center and ending at $5^{\prime}$ towards the north-east. More detailed variations are seen in the "plume", making it progressively hotter towards north-west. In the Fe abundance map, two zones are clearly distinct. There is an outer zone with Fe abundance of 0.1-0.2 solar and an inner zone, with Fe abundance in excess of 0.3 solar, the latter reaching 0.7 solar in some zones in the plume.

To estimate the pressure and entropy in each region, we need to estimate the length of the column for each selected two-dimensional region on the sky. We assumed a geometry, in which every region is part of a spherical shell that is centered on the core of M 86 system and has its inner and outer radii passing through the nearest and furthest points of the 

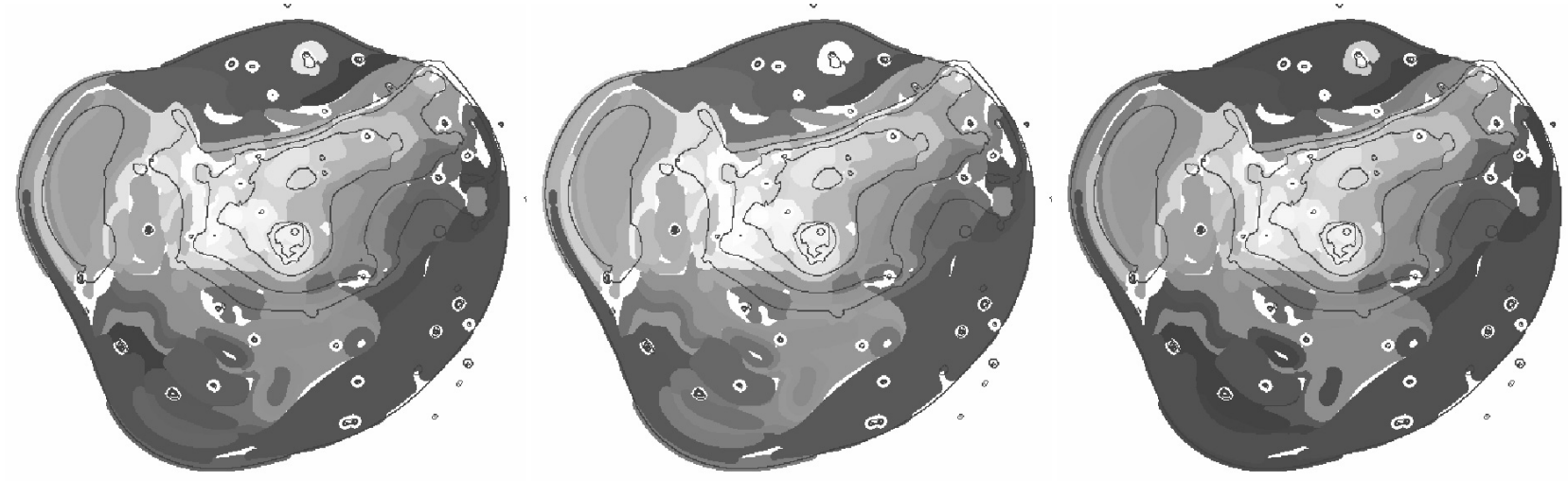

Fig. 3. Temperature map, overlaid with surface brightness contours. From left to right the panels show the best-fit values, low and upper limit on temperature, ranging from 0.6 (light grey) to 1.2 (dark grey) keV.
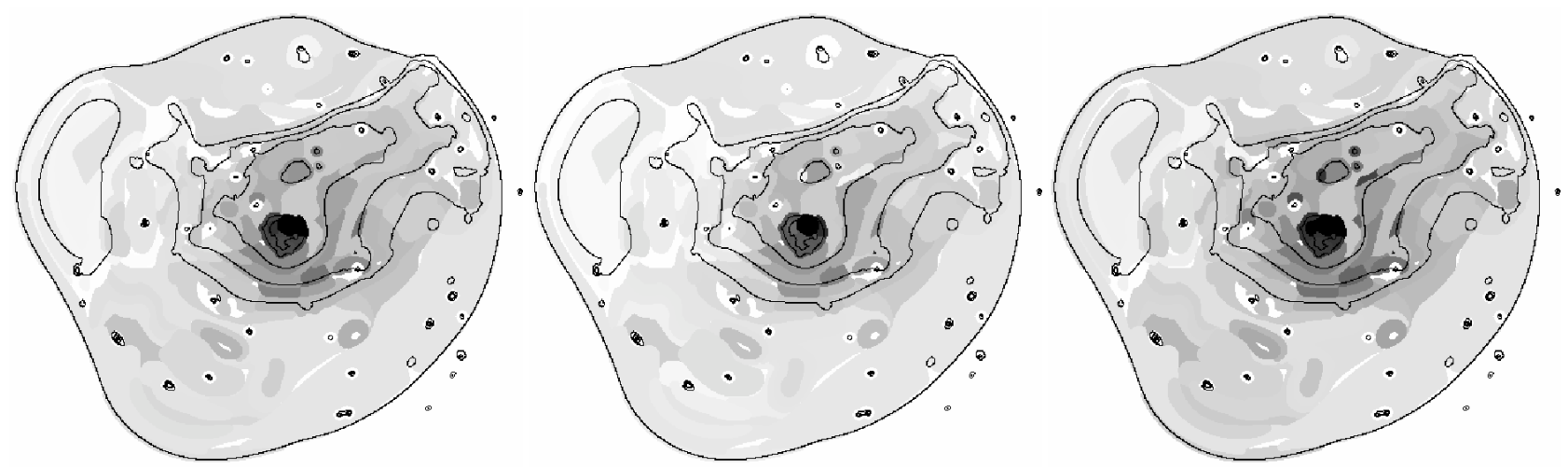

Fig. 4. Pressure map, overlaid with surface brightness contours. From left to right the panels show the best-fit values, low and upper limit on pressure, ranging from $2 \times 10^{-12} \mathrm{ergs} \mathrm{cm}^{-3}$ (light grey) to $2 \times 10^{-11} \mathrm{ergs} \mathrm{cm}^{-3}$ (black).
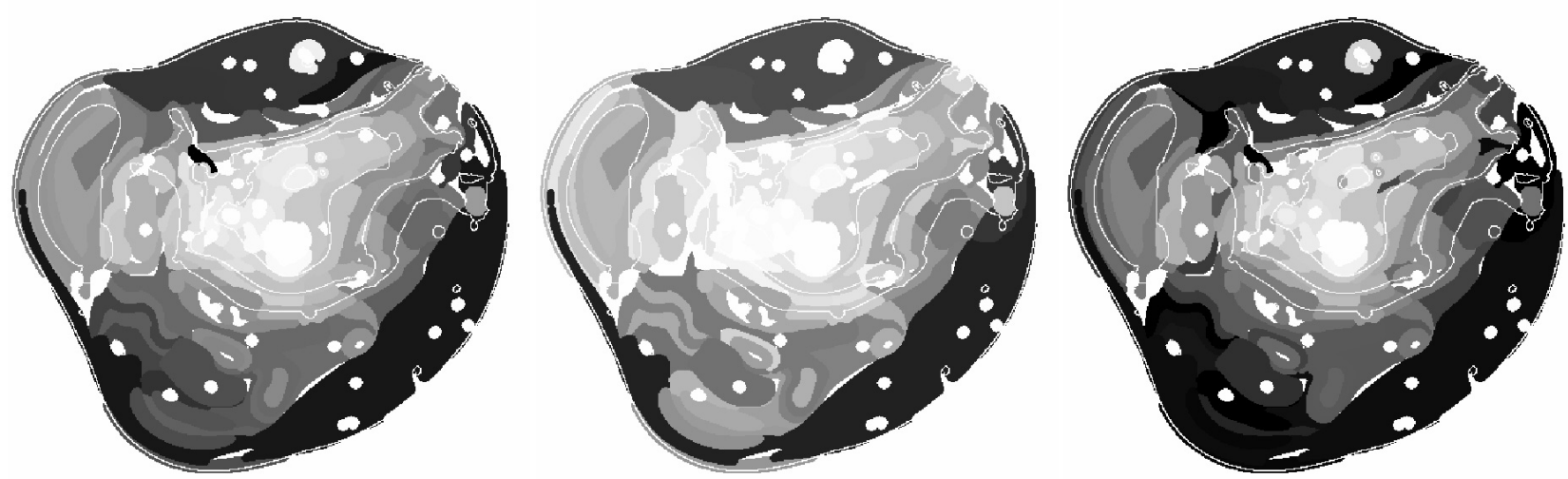

Fig. 5. Entropy map, overlaid with surface brightness contours. From left to right the panels show the best-fit values, low and upper limit on entropy, ranging from $8 \mathrm{keV} \mathrm{cm} \mathrm{cm}^{2}$ (white) to $100 \mathrm{keV} \mathrm{cm}^{2}$ (black).

selected region, respectively. This spherical shell is further intersected by a cylinder, that is directed towards the observer and in the observer plane has the cross-section of the selected region. For the concentric regions our geometry corresponds to a usual "onion peeling" technique (e.g. Finoguenov \& Ponman 1999). The advantage of our assumption is that the projected size depends only on the distance of the region to the center of the galaxy and not on the size of the extraction region. Alternatively, one could assume a projected dimention of the structure to be some combination of the observed dimentions. For large identified features, such as plume, north-eastern arm, and the shock, discussed below this makes less than $20 \%$ difference. Smaller features of such a geometry are unlikely to be detected, unless they correspond to an embedded low-entropy gas. For these we underestimate the pressure and overestimate the entropy by typically a factor of 1.3 . Using the adopted lineof-sight estimation method, in Figs. 4 and 5 we present the derived pressure and entropy maps. Note that we do not attempt to 


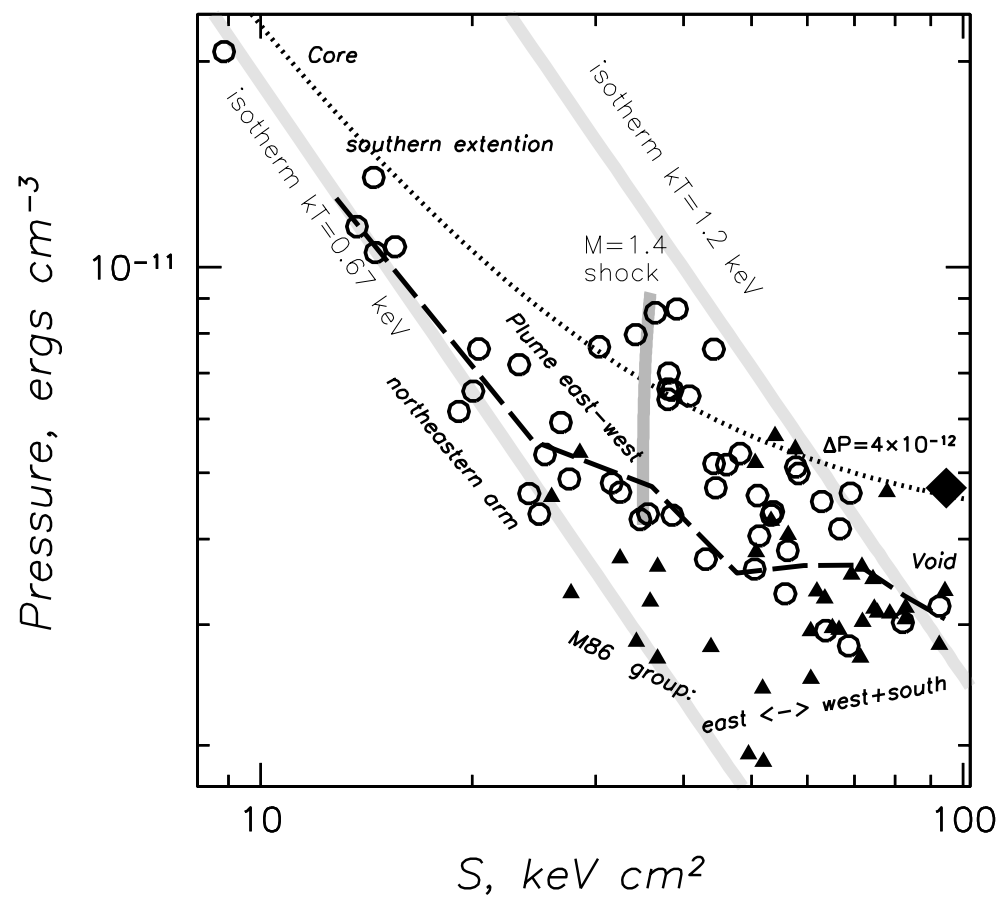

Fig. 6. Pressure vs. entropy for all the regions in M 86. Only the points with significance greater than 4 sigma are shown. Open circles and filled triangles denote the points with high and low Fe abundance, respectively. Light gray lines show isotherms and a dark-gray line shows the shock adiabat of Mach number of 1.4. Dotted line shows the prediction on imposing an additional pressure of $4 \times 10^{-12} \mathrm{ergs} \mathrm{cm}^{-3}$ to an initially isothermal gas with $k T=0.67 \mathrm{keV}$. Filled diamond displays the characteristics of the projected component found in the east to $\mathrm{M} 86$.

account for the effect outer components can have on the derived parameters of the inner components through the geometrical projection. However, projection is known to have a mild effect in X-ray systems with $\beta>0.5$, as is the case southwards from M 86 (Finoguenov \& Jones 2000), where we find the shock.

Low entropy zones include the core, part of the plume close to the core and the northeastern arm. Highest entropy regions form a circle surrounding M 86 system, suggesting that the large-scale emission of M 86 group is unperturbed. The pressure map also shows that the void region has a pressure typical of M 86 group, in agreement with a conclusion of Rangarajan et al. (1995), and it is rather M 86-galaxy that exhibits large contrast in the pressure map, which we analyze below.

\subsection{Revealing the shock}

In Fig. 6 we plot the pressure ( $n k T)$ vs. entropy $\left(k T n^{-\frac{2}{3}}\right)$ for all the regions for which the statistical errors on the pressure and entropy are less than $25 \%$. We assign the data to either M 86group (filled triangles) or M 86-galaxy (open circles), depending on whether the Fe abundance is above or below 1/4 solar, respectively. This threshold is higher than a typical element abundance for groups of 0.1 solar (e.g. Finoguenov et al. 2002), but allows a more robust criterion for intragroup media (IGM)ISM separation given the typical error on the iron abundance of 0.1 solar. This method is independent of geometry assumptions and it allows us to check whether or not such assumptions affect our derivation of thermodynamic quantities. We checked that using other elements leads to similar results, where statistics allows it.
In Fig. 6 we also show two isotherms, corresponding to $k T=0.67$ and $1.2 \mathrm{keV}$. Any uncertainty due to assumptions of geometry would move the data points only along isotherms. The high pressure, low entropy core is located on the top left corner of the plot. The plot shows that, as we move towards the outer regions of the galaxy the pressure drops while the entropy increases. Up to a few tens of $\mathrm{keV} \mathrm{cm}^{2}$, the selected regions are well aligned along a dashed line representing a massweighted mean of the data. We regard this behavior as typical as it compares well with ROSAT/ASCA results on other early-type galaxies from an extensive tabulation of Sanderson et al. (2003). Between 30 and $40 \mathrm{keV} \mathrm{cm}^{2}$ we notice that the data points suddenly appear distributed along two main loci with a gap in between. The origin of this could be ascribed either to a number of reasons such as enhanced data scatter and/or peculiar projection effects. However it could have a more interesting physical origin, specifically it could be due to the presence of a weak shock crossing M 86 medium. In fact the points along the two separate loci are well connected by a Rankine-Hugoniot shock adiabat corresponding to a Mach number shock of about 1.4. It is worth pointing out that a weak shock manifests primarily as a pressure jump and with only a minor entropy change as $\frac{\Delta S}{S} \propto\left(\frac{\Delta P}{P}\right)^{3}$, as it is the case of Fig. 6.

Additional support for our interpretation is provided by the spatial distribution of the points connected by the shock adiabat. This is shown in Fig. 7 where we display the regions containing the points with entropy between 30 and $40 \mathrm{keV} \mathrm{cm}$. Intriguingly, the regions (diagram points) associated with each of the two loci in the diagram, form two aligned, bow shaped and $180^{\circ}$ long strips, with the low pressure region closer to 


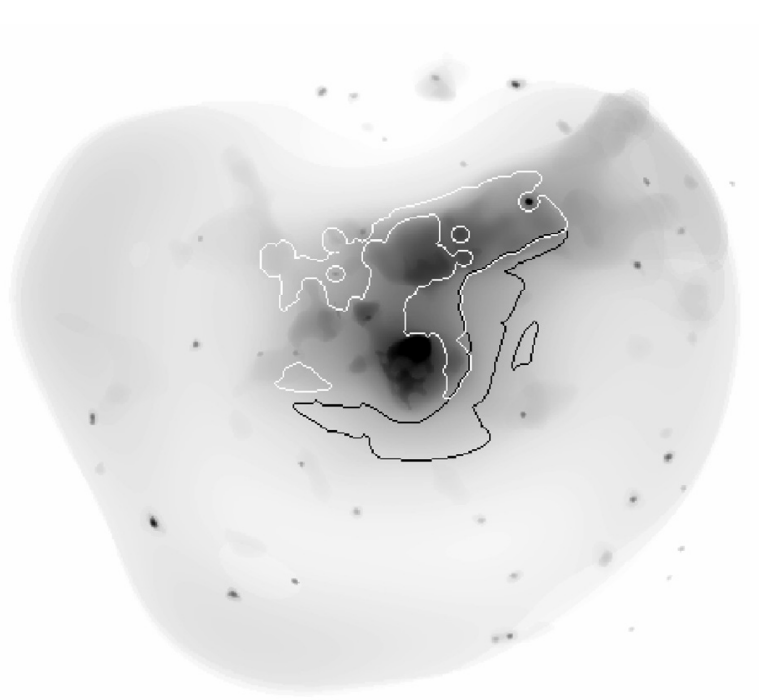

Fig. 7. Image of M 86. Black contours indicate shocked region, while white contours indicate the preshock regions of the same entropy and similarly high metallicity.

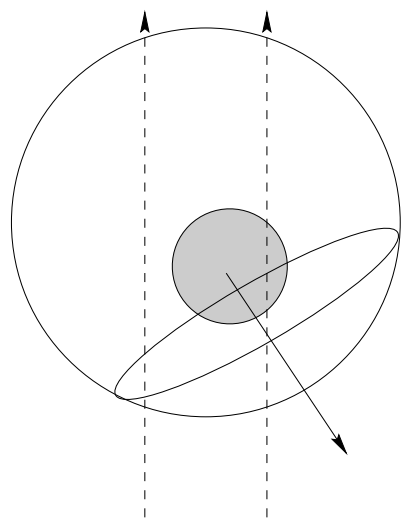

Fig. 8. Schematic view of the crossing of M 86 by the plane of the shock wave. Direction of motion of M 86 galaxy relative to the observer is indicated by a slight replacement of M 86 galaxy (filled circle) relative to the group (open circle).

M 86 core. Such pressure structure is clearly deviating from a hydrostatic profile. However, it is naturally interpreted in the framework in which a reverse shock is in the process of crushing M 86 ISM.

As with any study of this kind, unavoidable projection effects will produce some blending of pre- and post-shock regions reducing thus the estimate of the shock Mach number. A possible simplified geometry for the system M 86 cloud shock relative to an ideal observer is illustrated in Fig. 8. In this depiction the shock is oblique with respect to the line of sight. Only part of the shock is visible since most of the emission from the region to the north-east of M 86 is contributed by gas associated with the preshock material.

The passage of even a weak shock increases the pressure of the gas crossing through it. The shock weakens as is moves toward the inner, high pressure core of M 86 galaxy. In addition the pressure jump is lower in the outer regions where the

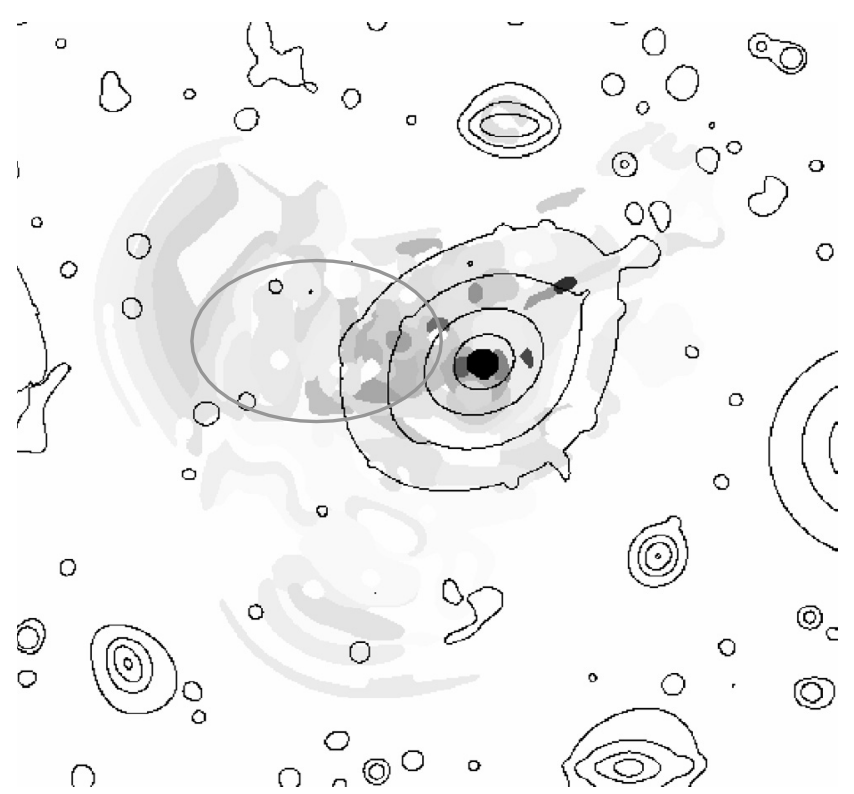

Fig. 9. Image of the intensity of the power law component per unit extraction area in the X-ray spectra of M 86. Contours indicate the surface brightness in the infrared, derived from DSS-2 data. Gray ellipse indicates the region selected for a detailed spectral analysis, presented in Fig. 10.

shock is oblique. Thus the region corresponding to an entropy of $30-40 \mathrm{keV} \mathrm{cm}^{-2}$ could represent the nose of the plunging gas cloud, and the higher entropy ones the outer zones where the shock induced pressure jumps are weaker. A maximum pressure increase of $4 \times 10^{12} \mathrm{ergs} \mathrm{cm}^{-3}$ is plotted in Fig. 6 to guide the eye.

\subsection{Additional emission components}

In the previous section we presented the results for the main emission component dominating the X-ray emission in the $0.5-2 \mathrm{keV}$ band. In addition we have introduced an underlying power law component, assuming it originates from unresolved point sources in M 86.

As such, the spatial distribution of this component should closely follow the distribution of the optical light of M 86 (e.g. Finoguenov \& Jones 2001). However, when we analyzed the distribution of the intensity of the power law component, a strong excess in the northeast was found, as illustrated in Fig. 9. Thus, we have found a disagreement with the assumption that the hard X-ray emission originates only due to LMXB. We have extracted a spectrum from the large region in the north-east to see in more detail the spectrum of the hard excess and realized that it is of thermal origin, having a temperature of $1.5 \pm 0.1 \mathrm{keV}$, as is shown in Fig. 10.

We have attempted an analysis with two temperature components, but the statistics is not good enough to derive any safe conclusion for our choice of the mask. In order to clearly separate this component one needs to group together the zones of similar temperature. This is exactly what has been done to extract this component. We believe this component corresponds to the shocked gas of M 86 seen in projection. Realistic 
Table 1. Properties of main regions of M 86.

\begin{tabular}{cccccccccccc}
\hline \hline $\mathrm{N}$ & Name & $\begin{array}{c}k T_{1} \\
\mathrm{keV}\end{array}$ & $\begin{array}{c}k T_{2} \\
\mathrm{keV}\end{array}$ & $\begin{array}{c}Z / Z_{\odot} \\
\end{array}$ & $\begin{array}{c}\text { norm } \\
10^{-3}\end{array}$ & $\begin{array}{c}\text { norm } \\
10^{-3}\end{array}$ & $\begin{array}{c}\chi^{2} \\
\text { d.o.f. }\end{array}$ & $\begin{array}{c}S \\
\mathrm{keV} \mathrm{cm} \mathrm{cm}^{2}\end{array}$ & $\begin{array}{c}P, 10^{-12} \\
\mathrm{ergs} \mathrm{cm}^{-3}\end{array}$ & $\begin{array}{c}M_{\mathrm{gas}} \\
10^{10} M_{\odot}\end{array}$ \\
\hline 01 & $\mathrm{NE} \mathrm{arm}$ & $0.690 \pm 0.013$ & $1.337 \pm 0.061$ & $0.64 \pm 0.04$ & $0.79 \pm 0.10$ & $0.41 \pm 0.07$ & 1.17 & 213 & $42 \pm 4$ & $5.3 \pm 0.3$ & $0.31 \pm 0.02$ \\
02 & core & $0.677 \pm 0.007$ & $1.188 \pm 0.047$ & $0.76 \pm 0.05$ & $0.41 \pm 0.05$ & $0.19 \pm 0.03$ & 1.31 & 171 & $14 \pm 1$ & $21.4 \pm 1.1$ & $0.03 \pm 0.01$ \\
03 & SE ext. & $0.659 \pm 0.020$ & $1.027 \pm 0.066$ & $0.56 \pm 0.05$ & $0.23 \pm 0.06$ & $0.14 \pm 0.05$ & 1.24 & 115 & $16 \pm 3$ & $15.1 \pm 1.7$ & $0.03 \pm 0.01$ \\
04 & shock: $\mathrm{W}$ & $0.831 \pm 0.005$ & $1.369 \pm 0.036$ & $0.98 \pm 0.04$ & $1.21 \pm 0.09$ & $1.40 \pm 0.10$ & 1.63 & 331 & $63 \pm 3$ & $4.0 \pm 0.1$ & $1.04 \pm 0.03$ \\
05 & east-1 & $0.741 \pm 0.018$ & $1.386 \pm 0.222$ & $0.29 \pm 0.03$ & $0.67 \pm 0.13$ & $0.44 \pm 0.12$ & 0.92 & 120 & $58 \pm 10$ & $3.8 \pm 0.5$ & $0.44 \pm 0.03$ \\
06 & shock: E & $1.605 \pm 0.141$ & $0.774 \pm 0.017$ & $0.67 \pm 0.09$ & $0.19 \pm 0.04$ & $0.17 \pm 0.04$ & 0.97 & 74 & $56 \pm 9$ & $6.0 \pm 0.6$ & $0.11 \pm 0.01$ \\
07 & plume & $0.792 \pm 0.004$ & $1.566 \pm 0.079$ & $1.18 \pm 0.06$ & $1.12 \pm 0.10$ & $0.44 \pm 0.06$ & 2.15 & 282 & $51 \pm 4$ & $5.3 \pm 0.2$ & $0.44 \pm 0.02$ \\
08 & trans. & $0.726 \pm 0.022$ & $1.278 \pm 0.073$ & $0.88 \pm 0.07$ & $0.29 \pm 0.06$ & $0.21 \pm 0.05$ & 1.16 & 163 & $26 \pm 4$ & $10.8 \pm 0.9$ & $0.07 \pm 0.01$ \\
09 & east-3 & $1.247 \pm 0.097$ & $0.666 \pm 0.062$ & $0.10 \pm 0.02$ & $1.04 \pm 0.20$ & $0.47 \pm 0.18$ & 1.14 & 101 & $59 \pm 10$ & $3.5 \pm 0.3$ & $0.61 \pm 0.06$ \\
10 & east-2 & $1.333 \pm 0.041$ & $0.666 \pm 0.020$ & $0.29 \pm 0.02$ & $1.21 \pm 0.14$ & $0.72 \pm 0.12$ & 1.10 & 199 & $65 \pm 6$ & $3.3 \pm 0.1$ & $0.86 \pm 0.04$ \\
11 & trans. & $0.811 \pm 0.041$ & $1.369 \pm 0.192$ & $0.36 \pm 0.06$ & $0.10 \pm 0.04$ & $0.17 \pm 0.04$ & 1.10 & 48 & $53 \pm 12$ & $5.5 \pm 0.7$ & $0.08 \pm 0.01$ \\
12 & trans. & $0.815 \pm 0.020$ & $1.512 \pm 0.146$ & $0.59 \pm 0.08$ & $0.14 \pm 0.04$ & $0.23 \pm 0.05$ & 1.48 & 63 & $56 \pm 9$ & $6.0 \pm 0.6$ & $0.11 \pm 0.01$ \\
13 & group SW & $1.483 \pm 0.027$ & $0.846 \pm 0.006$ & $0.44 \pm 0.01$ & $7.49 \pm 0.17$ & $2.30 \pm 0.16$ & 1.36 & 508 & $91 \pm 2$ & $3.2 \pm 0.1$ & $5.13 \pm 0.07$ \\
14 & Void & $1.234 \pm 0.022$ & - & $0.12 \pm 0.01$ & $2.82 \pm 0.14$ & - & 1.15 & 242 & $98 \pm 5$ & $2.7 \pm 0.1$ & $0.92 \pm 0.02$ \\
\hline
\end{tabular}

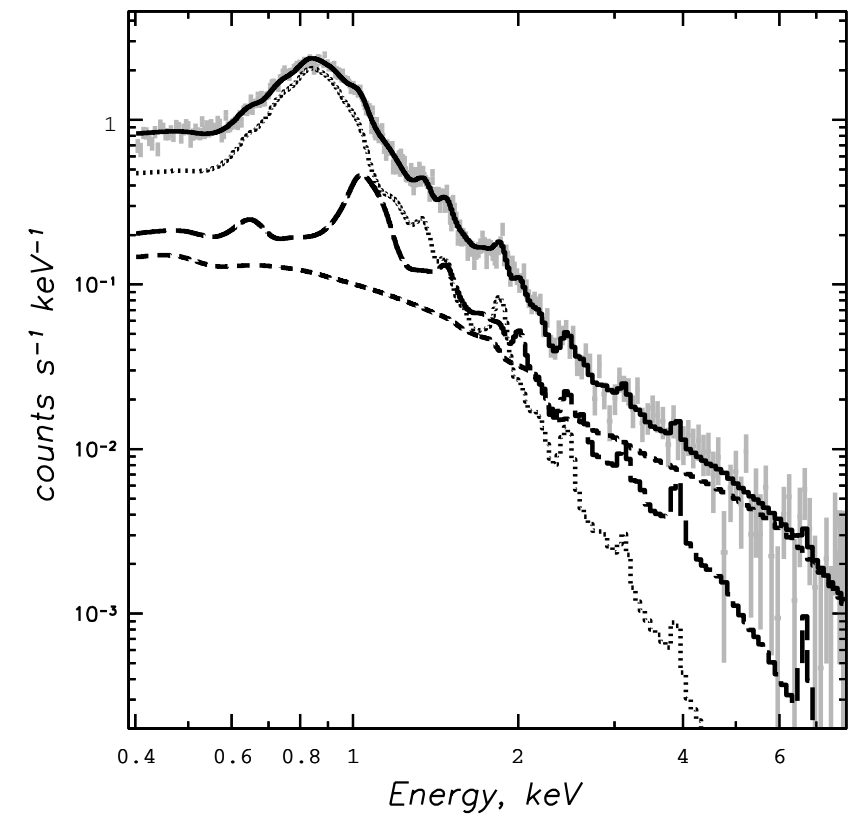

Fig. 10. Spectrum of the M 86 zone with enhanced hard emission. Grey crosses indicate the data points, solid line shows the combined model, consisting of normal component (dotted), hotter component (long-dashed) and a power-law (short-dashed).

placement of this component into Fig. 6, supports this scenario assigning this gas to M 86 group. We have checked the spectra south-east and south-west, they all are fitted by a $1.2 \mathrm{keV}$ temperature plasma, ruling out a possibility of a dense large-scale emission of $1.5 \mathrm{keV}$ temperature in front of M 86. Emission from the Virgo cluster is not contributing strongly within the XMM FOV.

\subsection{Main properties of $M 86$}

Once the detailed spectroscopic analysis has been performed, we could try to simplify the results by putting together all the regions of similar temperature. The minimum width of the region in this analysis is one arcminute, which also reduces possible influence of the point spread function on the analysis. Such region selection allows us to tabulate the basic properties of the M 86 ISM as well as take advantage of good statistics to look whether the spectral model adopted for the refined analysis is still a valid one. Instead of fitting the element abundance of all the elements, we use the photospheric solar abundance pattern of (Anders \& Grevesse 1989) and in Fig. 11 we demonstrate the quality of the fit. It becomes clear from this comparison that $\mathrm{O}$ is underabundant, while high energy lines, especially $\mathrm{Si} \mathrm{He}$-like triplet, is located on the blue side of the model, possibly implying an overcorrection for the pn CTI losses. Other than that, the adopted model provides a good description of the spectrum with parameters listed in Table 1. Column (1) presents the region label, as indicated in Fig. 1, Col. (2) lists the name of the region, Cols. (3 and 4) temperature of two thermal components, Col. (5) element abundance relative to the solar pattern of Anders \& Grevesse (1989), Cols. (6 and 7) normalizations of the two components in XSPEC units, Cols. ( 8 and 9) reduced $\chi^{2}$ and a number of degrees of freedom, which reach the finest possible energy binning for most spectra. Columns (10-12) present the entropy, pressure and gas mass of a sum of two components, assuming they occupy the same volume. Uncertainties in the parameter estimates are cited at the $68 \%$ confidence level and do not include any systematical uncertainties, either instrumental or model.

The mass-averaged properties of M 86 within the central $80 \mathrm{kpc}$ are $k T=1.17 \pm 0.02 \mathrm{keV}, F e=0.47 \pm 0.01 F e_{\odot}, S=$ $78 \pm 2 \mathrm{keV} \mathrm{cm}^{2}, P=(3.73 \pm 0.05) \times 10^{-12} \mathrm{ergs} \mathrm{cm}^{-3}$. The total gas mass is $(10.18 \pm 0.11) \times 10^{10} M_{\odot}$. The central $30 \mathrm{kpc}$ of M 86 are characterized by $k T=1.08 \pm 0.03, F e=0.95 \pm 0.03 F e_{\odot}$, $S=55 \pm 2 \mathrm{keV} \mathrm{cm}^{2}, P=5.33 \pm 0.11 \times 10^{-12} \mathrm{ergs} \mathrm{cm}^{-3}$, massaveraged over the total gas mass of $(2.03 \pm 0.04) \times 10^{10} M_{\odot}$. As no account for the gas mass not associated with the directly observed component was attempted, the total gas mass reported here is a factor of two smaller than the gas mass enclosed within the radius of observation. 

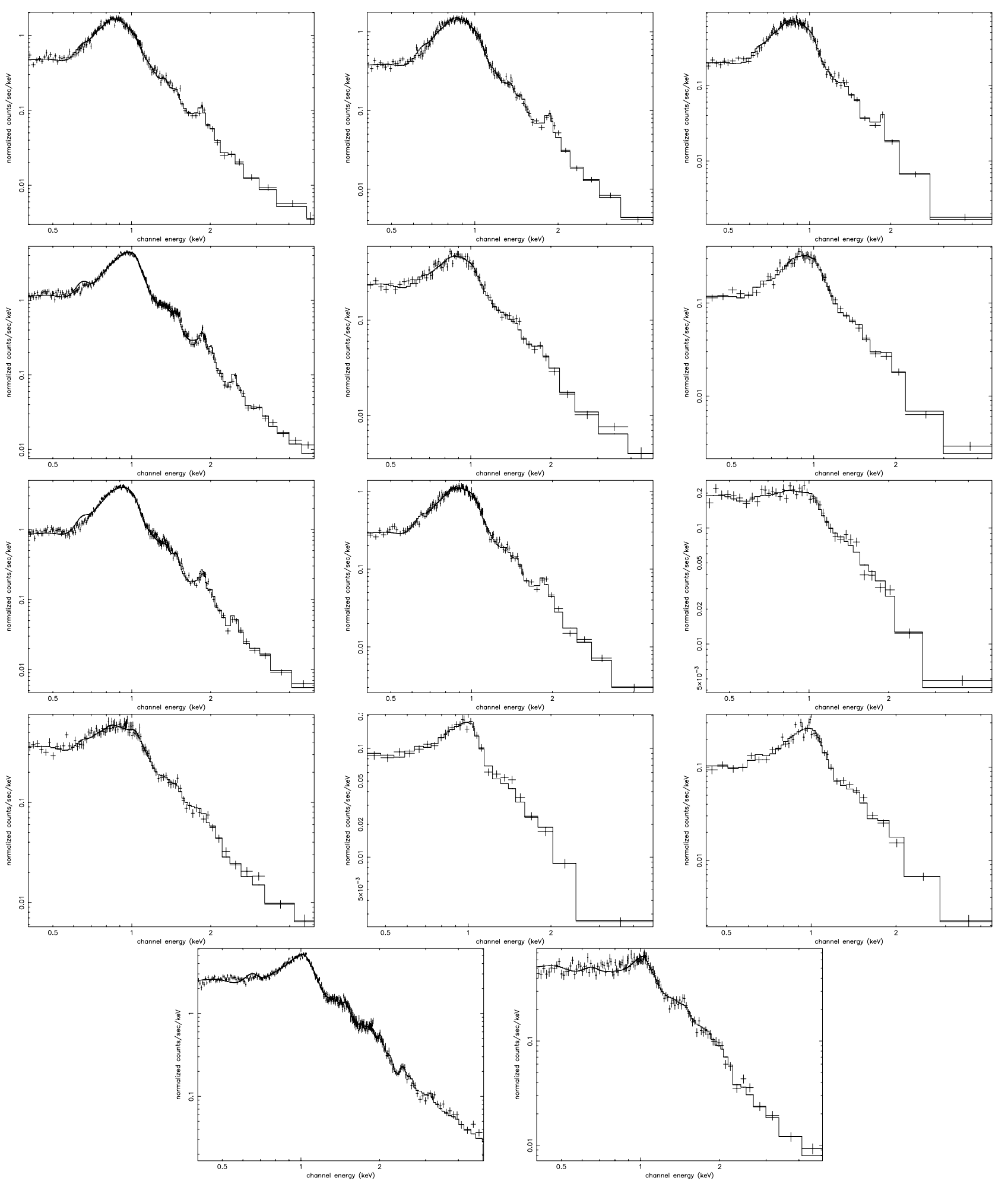

Fig. 11. Spectra of 14 major zones of the M 86, in correspondence with Table 1. Spectra are assembled in rows with increasing number from left to right and from up to down.

\section{Discussion}

XMM-Newton observations presented here, while confirming previous arguments in favor of strong interactions taking place in M 86, provide new insights as to their yet unclear nature. As already mentioned in the introduction previous works concentrated on M 86 galaxy interaction with Virgo's ICM. However, a number of observations challenge this picture: the distance of $2.4 \pm 1.4 \mathrm{Mpc}$ between the M 86 and M 87 (Neilsen \& Tsvetanov 2000). This result implies that M 86 lies outside the virial radius of the Virgo cluster. Also, based on the RASS surface brightness measurements (Böhringer et al. 1994) the Xray emission surrounding the M 86 galaxy is most naturally 


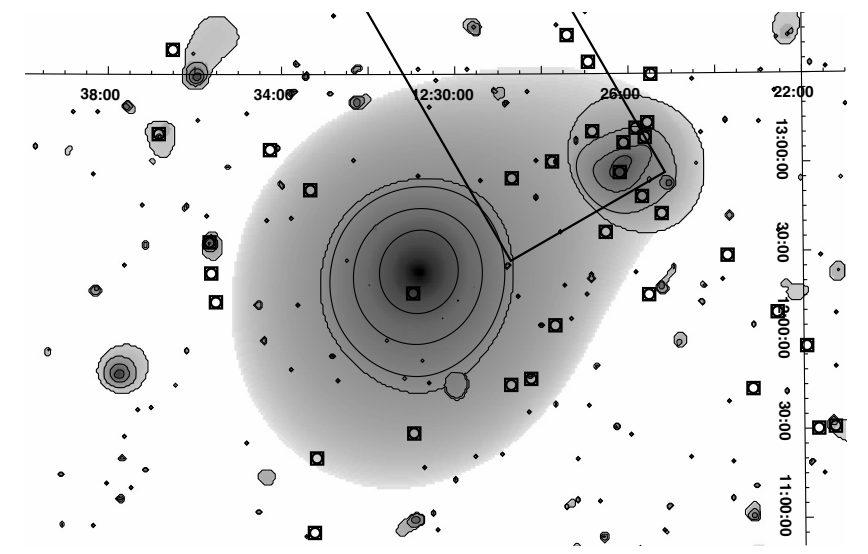

Fig. 12. Large-scale structure around $M 86$. The wavelet-reconstructed image reproduces a part of ROSAT All-Sky Survey in the $0.5-2 \mathrm{keV}$ energy band, close to $\mathrm{M} 86$. Black squares indicate the galaxies falling onto Virgo from behind $\left(V_{\mathrm{M} 87}-V_{\text {galaxy }}>600 \mathrm{~km} \mathrm{~s}^{-1}\right)$, using the galaxy velocity data from Bingelli et al. (1985, 1993). Large rectangular region denotes the position of the excess X-ray emission identified in Böhringer et al. (1994).

associated to M 86's group medium, as opposed to Virgo ICM as often assumed in previous studies. XMM-Newton provides an additional proof to this picture by measuring the $1.2 \mathrm{keV}$ temperature of the group component, compared to $2.4 \mathrm{keV}$ temperature expected from the Virgo cluster.

Although we dismiss Virgo ICM (more correctly, M 87 cloud) as responsible for M 86 X-ray morphological disruption, obviously our depiction still requires the presence of a dense medium for M 86 to interact with. We assume for the sake of simplicity that M 86 velocity with respect to the putative medium is the same as that relative to $M 87$, that is about $1500 \mathrm{~km} \mathrm{~s}^{-1}$. Then we find that a medium number density no less than a few $\times 10^{-4}$ is required in order for the observed postshock pressure of order $5 \times 10^{-12} \mathrm{ergs} \mathrm{cm}^{-3}$ to be generated. Such diffuse gas should be detectable in X-ray emission if it had a temperature in excess of $0.2 \mathrm{keV}$. In fact, Böhringer et al. (1994, see their Fig. 2) had already pointed out the presence of a large-scale X-ray filament in precisely that region. It was revealed in the RASS data as residual emission after removal of the contributions associated with both M 87 and M 86. The density of such structure was typically found about $10^{-3} \mathrm{~cm}^{-3}$, sufficiently high to justify the interpretation presented above on M 86 dynamics. Its width (on the plane of the sky) is about $300 \mathrm{kpc}$, typical for a filament, yet the high density suggests a high degree of virialization such as that characteristic of a chain of groups. Figure 12 summarizes the proposed interaction geometry. We note that both the X-ray emission and infalling galaxies are present at the position of M 86. It is remarkable that the alignment of the residual X-ray emission is parallel to the suggested direction of motion of M 86 in the observer plane to the south-west.

Presence of low-entropy tails behind the M 86 core, such as plume and northeastern arm strongly suggest that M 86 has already been harassed, prior to the generation current shock wave. Quite strikingly the large-scale emission morphology of M 86 group displayed in Fig. 12 appears relaxed, while strong interactions are taking place in the core of M 86 galaxy. This suggests that the interactions responsible for the morphological disturbances in M 86 galaxy were produced by objects which did not affect significantly the gas in M 86 group. One such possibility is provided by high velocity galaxy-galaxy encounters. Therefore, M 86 system could provide an important case for establishing the morphology-density relation between one and two virial radii, as suggested by the SDSS results (e.g. Goto et al. 2003).

The possibility of galaxy-galaxy interactions prior to infall onto a cluster is not remote. M 86 group appears to be attracted toward the Virgo supercluster with mass roughly $\sim 10^{15} M_{\odot}$ (see also Neilsen \& Tsvetanov 2000). Numerous other galaxies accreting from different distances will have different accretion velocities as well as directions allowing for a high-speed interactions to take place. Most observers agree that the direction of the motion of M 86 in the observer's plane is towards the south with some component to the west. So, M 86 is aiming to the point of interaction of M 87 and M 49, but slightly bears off west. Another dozen of infalling galaxies, displayed in Fig. 12 seems to share the same route and some of them could be the former collision companions to M 86. The magnitude of the interaction, recorded in M 86 X-ray image, should have had a strong effect on those galaxies, leading to substantial depletion in the HI content. Our estimate of the ram pressure to be of the order of $4 \times 10^{-12} \mathrm{ergs} \mathrm{cm}^{-3}$ is similar to ram pressure expected to act on galaxies within the Virgo cluster, as described in Vollmer et al. (2001).

\section{Conclusions}

An in-depth understanding of the processes in the hot interstellar medium of M 86, provided by observations of M 86 has suggested a number of past and on-going interactions to take place. Comparison with the position of M 86 in the Virgo cluster, promotes the environment outside the virial radius of M 87 cloud to bear the prime responsibility for the apparent morphological transformation of the X-ray appearance of M 86. Some of the past interactions in M 86 are characterized by $10-50 \mathrm{kpc}$ scale, which is only suitable for galaxy-galaxy interactions on one hand, setting limits on a degree of interaction, on the other, thus providing us with understanding of the processes leading to establishment of the morphology-density relations in clusters of galaxies.

Acknowledgements. This paper is based on observations obtained with XMM-Newton, an ESA science mission with instruments and contributions directly funded by ESA Member States and the USA (NASA). The XMM-Newton project is supported by the Bundesministerium für Bildung und Forschung/Deutsches Zentrum für Luft- und Raumfahrt (BMFT/DLR), the Max-Planck Society and the Heidenhain-Stiftung, and also by PPARC, CEA, CNES, and ASI. A.F. acknowledges receiving the Max-Plank-Gesellschaft Fellowship. F.M. was partially supported by the European Community through the Research and Training Network - "The Physics of the Intergalactic Medium" - under contract HPRN-CT2000-00126 RG29185. A.F. acknowledges useful discussions with Hans Böhringer, Bernd Vollmer, Jacqueline van Gorkom and Cristina Popescu. The authors thank the anonymous referee for useful criticism. 


\section{References}

Anders, E., \& Grevesse, N. 1989, Geochim. Cosmochim. Acta, 53, 197

Binggeli, B., Sandage, A., \& Tammann, G. A. 1985, AJ, 90, 1681

Binggeli, B., Popescu, C. C., \& Tammann, G. A. 1993, A\&AS, 98, 275

Böhringer, H., Briel, U. G., Schwarz, R. A., et al. 1994, Nature, 368, 828

Briel, U. G., Finoguenov, A., \& Henry, J. P. 2003, A\&A, in preparation

Fabian, A. C., Schwarz, J., \& Forman, W. 1980, MNRAS, 192, 135

Finoguenov, A., \& Ponman, T. J. 1999, MNRAS, 305, 325

Finoguenov, A., \& Jones, C. 2000, ApJ, 539, 603

Finoguenov, A., \& Jones, C. 2001, ApJ, 547, 107

Finoguenov, A., \& Jones, C. 2002, ApJ, 574, 754

Finoguenov, A., Jones, C., Böhringer, H., \& Ponman, T. J. 2002, ApJ, 578,74

Forman, W., Schwarz, J., Jones, C., Liller, W., \& Fabian, A. 1979, ApJ, 234, L27

Goto, T., Okamura, S., Sekiguchi, M., et al. 2003, PASJ, 55, 757

Irwin, J. A., Athey, A. E., \& Bregman, J. N. 2003, ApJ, 587, 356

Jansen, F., Lumb, D., Altieri, B., et al. 2001, A\&A, 365, L1

Liedahl, D. A., Osterheld, A. L., \& Goldstein, W. H. 1995, ApJ, 438, L115
Lumb, D. H., Finoguenov, A., Saxton, R., et al. 2003, SPIE, 4851, 255

Kodama, T., Smail, I., Nakata, F., Okamura, S., \& Bower, R. G. 2001, ApJ, 562, L9

Neilsen, E. H., Jr., \& Tsvetanov, Z. I. 2000, ApJ, 536, 255

Poggianti, B., Smail, I., Dressler, A., et al. 1999, ApJ, 518, 576

Rangarajan, F. V. N., White, D. A., Ebeling, H., \& Fabian, A. C. 1995, MNRAS, 227, 104

Raymond, J., \& Smith, B. 1977, ApJS, 35, 419

Read, A. M. 2003, preprint [astro-ph/0212436]

Sanderson, A. J. R., Ponman, T. J., Finoguenov, A., Lloyd-Davies, E. J., \& Markevitch, M. 2003, MNRAS, 340, 989

Smith, R. K., Brickhouse, N. S., Liedahl, D. A., \& Raymond, J. C. 2001, ApJ, 556, L91

Stark, A. A., Gammie, C. F., Wilson, R. W., et al. 1992, ApJS, 79, 77

Strüder, L., Briel, U. G., Dennerl, K., et al. 2001, A\&A, 365, L18

Takeda, H., Nulsen, P. E. J., \& Fabian, A. C. 1984, MNRAS, 208, 261

Turner, M. J. L., Abbey, A., Arnaud, M., et al. 2001, A\&A 365, L27

Vikhlinin, A., McNamara, B. R., Forman, W., et al. 1998, ApJ, 502, 558

Vikhlinin, A., Markevitch, M., \& Murray, S. S. 2001, ApJ, 551, 160

Vollmer, B., Cayatte, V., Balkowski, C., \& Duschl, W. J. 2001, ApJ, 561,708 